\title{
Bandwidth Enhancement of Symmetrical Fourth-Teeth-Shaped Microstrip Antenna
}

\author{
Angelina G. Markina1, Dmitrii N. Tumakov², Nikolai B. Pleshchinskii ${ }^{3}$ \\ 1,2, ${ }^{3}$ Kazan Federal University \\ Email: tdn2003@list.ru, Contact: +7 (965) 5867997
}

Received: 21st October 2017 Accepted: 16th November 2017, Published: 31st December 2017

\begin{abstract}
The microstrip antenna with a symmetrical rectangular radiator and four teeth is described. The influence of the base geometric parameters of the antenna on the bandwidth at the base frequency was studied. The following geometric parameters of the antenna are selected: the length and the width of the radiator, the depth of cuts, the thickness of the substrate, the length of the ground plane and the width of the feed line. The regression analysis was carried out and the mathematical model describing the dependence of the bandwidth on the length and the width of the radiator and the depth of the cuts was developed. The rootmean-square error and the relative absolute error of the model were calculated. The graphs of the bandwidth dependences on the geometric parameters are presented.

It was established that the decrease of the bandwidth values is associated with an increase of the radiator width and the substrate thickness. It was shown that a slight influence on the bandwidth are made by the changes of the radiator length and the depths of the cuts only in the case when the radiator width is much smaller than its length. The proposed formula describing the relationship of the bandwidth with the geometric parameters of the antenna can be used to design a four-tooth antenna with wide bandwidth.
\end{abstract}

Key words: Bandwidth, Microstrip Antenna, FourthTeeth-Shaped Antenna, Bandwidth Enhancement.

\section{Introduction}

Nowadays microstrip antennas are among the most common and widely used types of antennas [1]. The most studied of them are microstrip antennas with rectangular and other radiators of simple geometry $[1$, 2]. However, the narrow bandwidth restricts the use of such antennas. There are various methods for this problem solution [3-6]. The bandwidth can be widened by adding the cutouts to the radiator. For example, the slots in the form of the letter L [7], H and $\mathrm{U}$ [8], the letter E [9] and even their combinations [10] are used. The ground plane is changed [11-14], as well as other methods [15, 16] are used, including the use of metamaterials [17, 18]. Due to these and other optimization approaches, the antennas not only increase the bandwidth, but also improve other characteristics of the antennas.
One of the promising areas is the creation of so-called tooth antennas (the antennas with the cutouts on the sides of a rectangular radiator). For example, in [19] such an antenna is described with the cutouts only on one side, and the symmetrical tooth antenna was studied in [20]. In [21], it is considered tooth antennas with stepped radiating elements, and microstrip patch antenna with seven operating ranges is presented in [22].

However, the process of any antenna design that has certain electrodynamic characteristics is quite long and time consuming. One of the promising approaches is the use of regression models describing the relationship between the electrodynamic characteristics of an antenna and its geometry [23]. Using these relationships, you can determine an approximate shape of an antenna immediately, which will be well matched in a given frequency range. This approach facilitates the antenna design process and allows to obtain a wellmatched antenna in a shorter period of time.

In this paper we consider the antenna with a symmetrical four-tooth radiator. The influence of the radiator geometry on the antenna bandwidth is shown. The regression model is designed that describes the relationship of the bandwidth at the base frequency with the geometric parameters of the radiator. The influence of the substrate thickness, ground plane dimensions, radiator scale and the width of the feeding line on the bandwidth are analyzed. Graphs showing the dependence of the bandwidth on tunable antenna parameters are presented.

\section{Problem Statement}

Let us consider a microstrip antenna with a radiator of a symmetric four-comb shape (Fig. 1). The front side of $a_{S} \times b_{S}$ substrate with the dielectric permittivity $\varepsilon_{r}=$ 4.5 , the material density $\rho=1000 \mathrm{~kg} / \mathrm{m}^{3}$ and the tangent of the dielectric loss angle $\operatorname{tg} \sigma=0$ has the radiator of $a_{R} \times b_{R}$ with a straight feeding line. The width $w_{F}$ and the length $l_{F}$ of the feed line $(50 \Omega)$ will be $1 \mathrm{~mm}$ and $15 \mathrm{~mm}$ respectively. The back side of the substrate will have the ground plane with the length of $b_{G}$ and the width for the entire dielectric, and we assume that $b_{G}=l_{F}$. The thickness of the substrate is set equal to $1 \mathrm{~mm}$, and the dimensions $a_{S}$ and $b_{S}$ are set equal to $30 \mathrm{~mm}$ and $75 \mathrm{~mm}$, respectively.

Let us make symmetrical rectangular slots with the depth $d_{R}$ on the two sides of the radiator (left and right), 
and the comb width of the radiator $c_{R}$ will be determined according to the formula $c_{R}=b_{R} / 3$.

Let us study the influence of the radiator geometric parameters $\left(a_{R}, b_{R}\right.$ and $\left.d_{R}\right)$ on the bandwidth at the base frequency $\left(S_{11}<-5 \mathrm{~dB}\right)$. Let us perform the regression analysis and design a functional dependence of the antenna bandwidth on the radiator parameters.

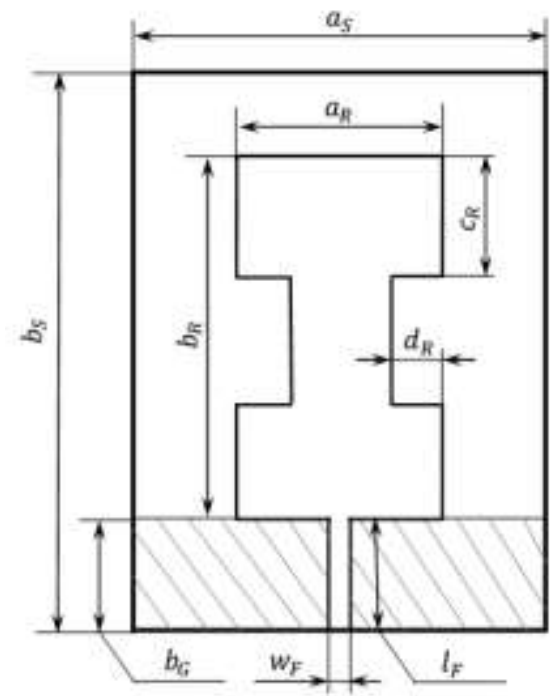

Figure 1. Four-Comb Shaped Microstrip Antenna

Let us first carry out numerical experiments for regression analysis. In the experiments the values $b_{R}$ of the radiator length will vary from 24 to $41 \mathrm{~mm}, a_{R}$ values of the radiator width will vary from 10 to 24 $\mathrm{mm}$, and the values $d_{R}$ of the slot depth will vary from 0.5 to $11 \mathrm{~mm}$ (depending on the radiator width). The total number of considered antennas is 204 .

\section{Dependence of the Bandwidth on the Radiator Parameters}

Let us consider the dependence of the bandwidth at the base frequency on the depth of cuts, the width and the length of the radiator. Let us demonstrate on Fig. 2-4 $\mathrm{BW}, \mathrm{GHz}$

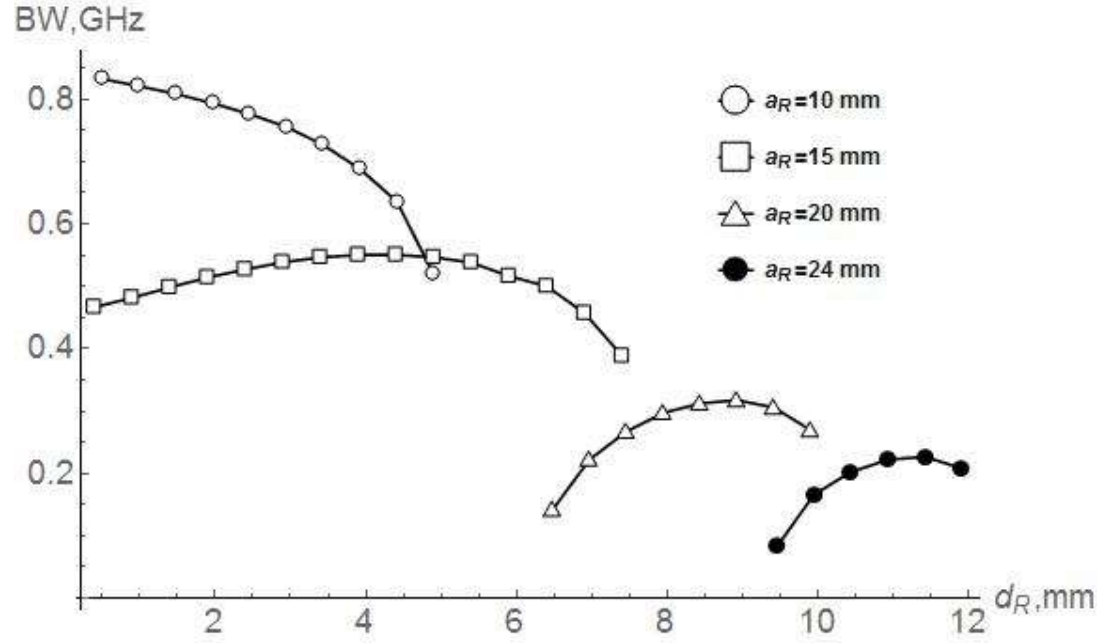

Figure 2. Dependence of $B W$ on the Cuts Depth $d_{R}$ for Different Radiator Width $a_{R}$ and the Radiator Length $b_{R}=24$ mm

Fig. 3 shows the dependence of the bandwidth on the size of the cuts at the radiator length $b_{R}=32.5 \mathrm{~mm}$. For narrow radiators $a_{R}=10 \mathrm{~mm}$ and $a_{R}=15 \mathrm{~mm}$, the decrease of $B W$ values is observed, and for $a_{R}=10$ the dependencies the bandwidth on the depth of cuts $d_{R}$ and the width of the radiator $a_{R}$ for fixed lengths of the radiator $b_{R}=24$, of 32.5 and $41 \mathrm{~mm}$.

Fig. 2 shows that the bandwidth values decrease with the increasing width of the radiator. The increase in the size of the cutouts for different widths of the radiator affects the bandwidth in different ways. For the narrow radiator $a_{R}=10 \mathrm{~mm}$, the bandwidth values decrease. For the radiator with $a_{R}=15 \mathrm{~mm}$, the $B W$ values increase slightly and then decrease. For the radiators whose the length and the width are close $\left(a_{R}=20 \mathrm{~mm}\right.$ and $a_{R}=24 \mathrm{~mm}$ ), we have a more pronounced increase and decrease of the bandwidth at large cuts sizes. 
Helix Vol. 8(1): 2275 - 2283

of $B W$ values, i.e. the increase and the subsequent

decrease of the bandwidth.

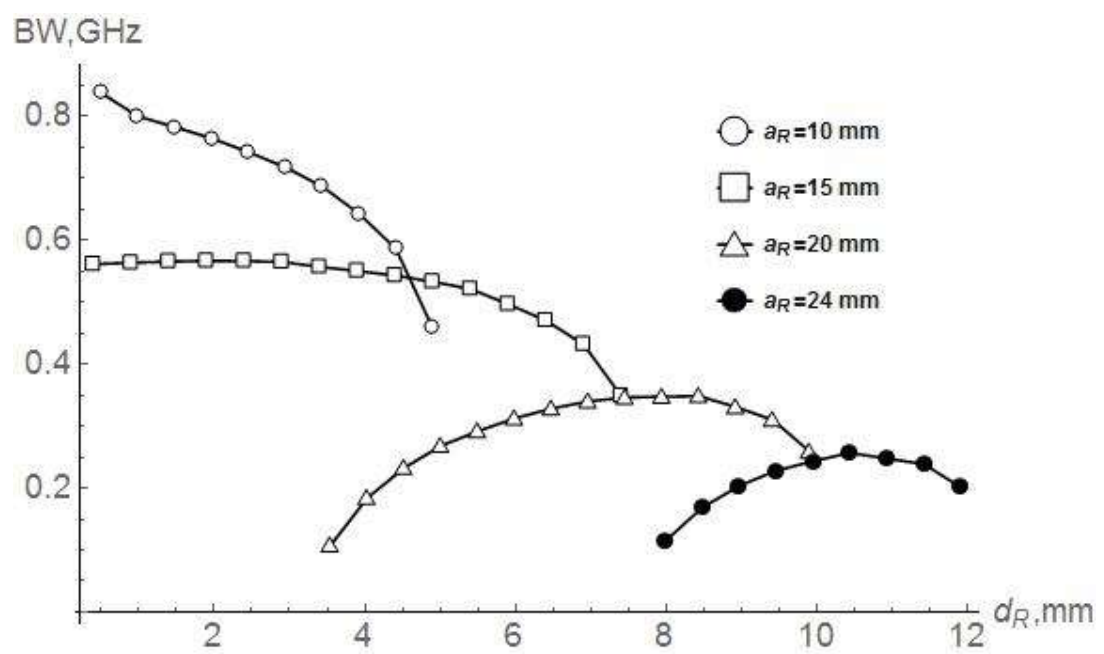

Figure 3. Dependence of $B W$ on the Cuts Depth $d_{R}$ for Different Radiator Width $a_{R}$ and the Radiator Length $b_{R}$ $=32.5 \mathrm{~mm}$

Fig. 4 shows the dependence of the bandwidth on the cutouts size at the length of the radiator $b_{R}=41 \mathrm{~mm}$. The behavior of $B W$ values changes is similar to other

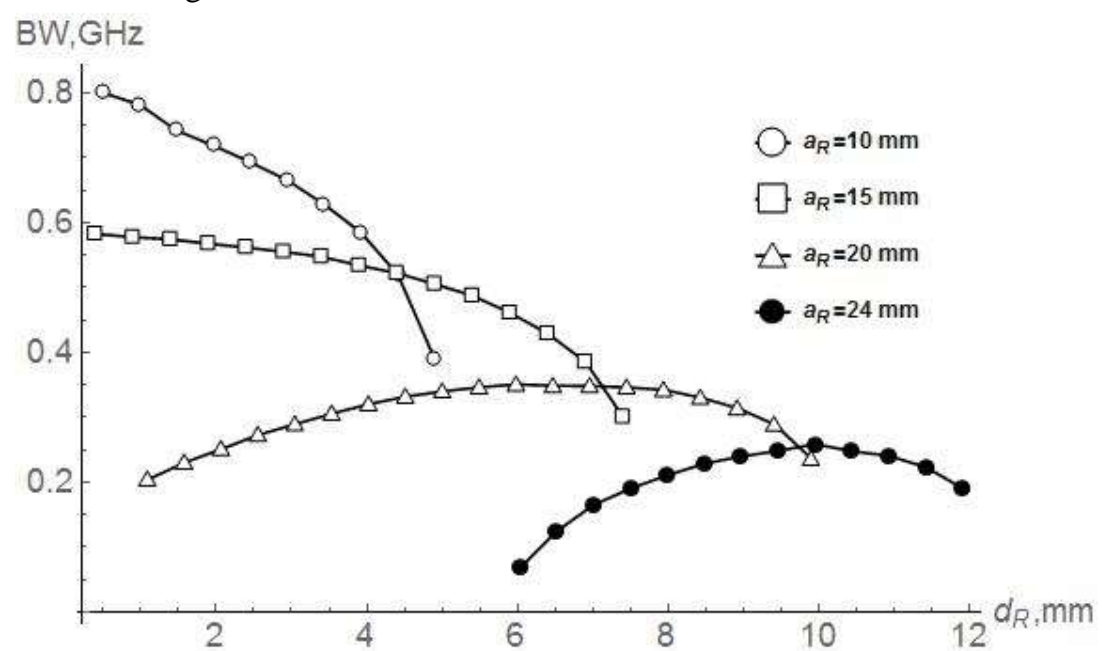

Figure 4. Dependence of $B W$ on the Cuts Depth $d_{R}$ for Different Radiator Width $a_{R}$ and the Radiator Length $b_{R}=41$ $m m$

For a more complete understanding the relationship between $B W$ and the radiator parameters, let us
$b_{R}$. The small difference lies in the fact that the bandwidth with the increase of the slot size is characterized by a large range of $B W$ value change.

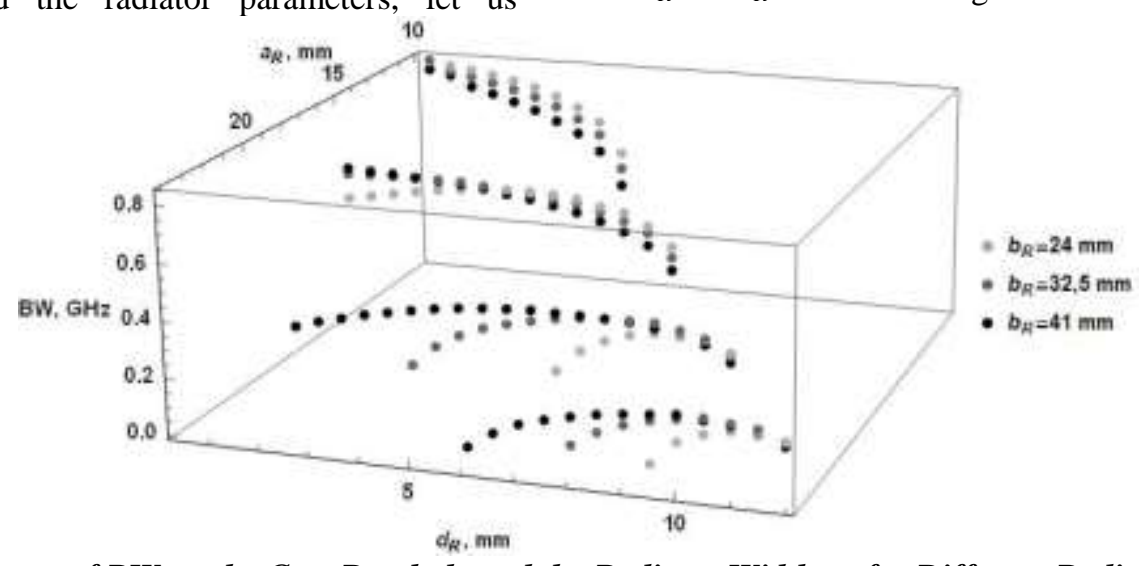

Figure 5. Dependence of $B W$ on the Cuts Depth $d_{R}$ and the Radiator Width $a_{R}$ for Different Radiator Length $b_{R}$ 
The graphs show clearly that the values of $B W$ decrease with increasing width of the radiator. Here each group of curves, consisting of three lines for different $b_{R}$ values is characterized by a smaller bandwidth. For similar curves in different groups, one can note their close $B W$ values and a similar behavior.

The general analysis of the graphs presented on Fig. 25 , allows us to conclude that the bandwidth is less dependent on the radiator length and depends more on the radiator width and on the values of $a_{R}-d_{R}$. Let us note that the values of $B W$ in different planes form an inverted parabola, and the saddle surface in space. It is also necessary to take into account the sharp decrease of $B W$ values as $d_{R}$ values approach $a_{R} / 2$.

Thus, the regression model $B W$ at the base frequency will be designed in the following form:

$$
\overline{B W}=C_{1} a_{R}+C_{2} a_{R}^{2}+C_{3} \frac{\left(a_{R}-d_{R}\right)}{b_{R}}+C_{5} \frac{\left(a_{R}-d_{R}\right)^{2}}{b_{R}}+C_{4} \ln \left(a_{R}-d_{R}\right),
$$

where $B W$ is measured in $\mathrm{GHz}$, and the length $b_{R}$ of the radiator, the width $a_{R}$ of the radiator and the depth of cuts $d_{R}$ are measured in $\mathrm{mm}$, and the coefficients $C_{m}$ are assumed to be unknown.
Using the method of least squares, we define the unknown parameters in (1). Then the required functional dependence for the bandwidth will have the following final form:

$$
\begin{aligned}
& \overline{B W}=-0.126464 a_{R}+0.00228148 a_{R}^{2}+1.78408 \frac{\left(a_{R}-d_{R}\right)}{b_{R}} \\
& -0.161612 \frac{\left(a_{R}-d_{R}\right)^{2}}{b_{R}}+0.807302 \ln \left(a_{R}-d_{R}\right) .
\end{aligned}
$$

Let us calculate the mean square error [24]:

$$
R M S E=\sqrt{\frac{1}{n} \sum_{i=1}^{n}\left(B W_{i}-\overline{B W}_{i}\right)^{2}}
$$

and the relative absolute error [24]:

$$
\sigma=\frac{1}{n} \sum_{i=1}^{n}\left|\frac{B W_{i}-\overline{B W}_{i}}{B W_{i}}\right| \cdot 100 \%
$$

where $B W_{i}$ are known values, and $\overline{B W}_{i}$ are the values calculated by the formula (2). For the regression model (2): $\mathrm{RMSE} \approx 0.03 \mathrm{GHz}, \sigma \approx 7.44 \%$. The formula (2) can be used to find the maximum $B W$ values at given constraints on the radiator parameters.

\section{Influence of the other Antenna Parameters on the Bandwidth}

Let us study the effect of other geometric parameters of the radiator: the length of the ground plane, the size of the radiator, the thickness of the substrate and the width of the feed line on the bandwidth of the fourteeth-shaped microstrip antenna. For comparative analysis, we select two antennas with the radiator size $a_{R}=10 \mathrm{~mm}$ at $b_{R}=24 \mathrm{~mm}$ and the depth of the cuts $d_{R}$ $=0.5 \mathrm{~mm}$ and $d_{R}=2.5 \mathrm{~mm}$. The remaining parameters of the antennas are assumed to be the same as in paragraph 2 .

At first, let us consider the effect of the ground plane size on the bandwidth. Fig. 6 shows the dependence of bandwidth on the length of the ground plane $b_{G}$. The graph with black triangles corresponds to the antenna with the depth of cuts $d_{R}=2.5 \mathrm{~mm}$, the graph with white triangles corresponds to the antenna with $d_{R}=$ $0.5 \mathrm{~mm}$. 


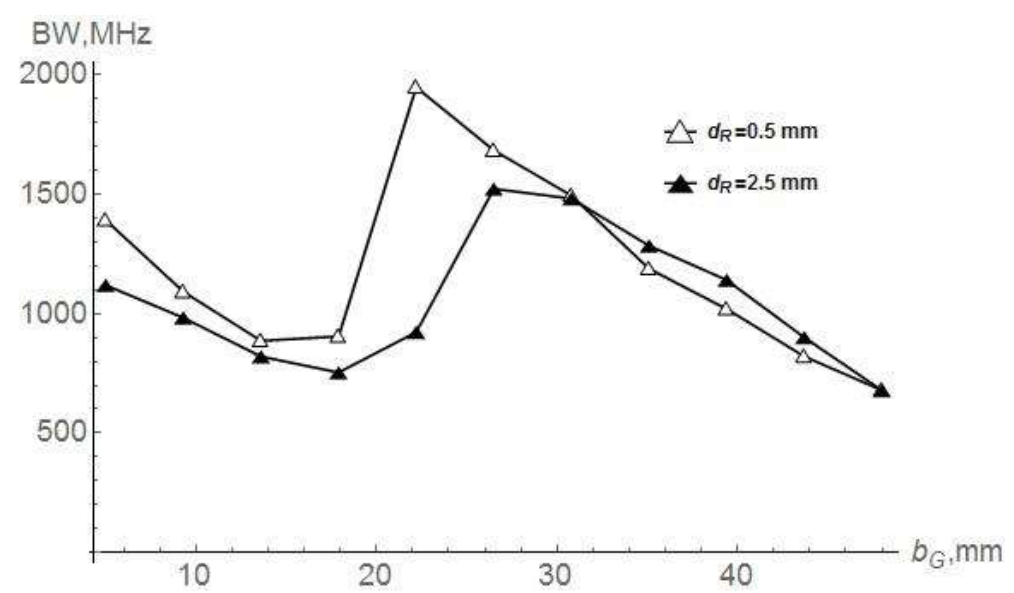

Figure 6. Dependence of $B W$ on the Length of Ground Plane $b_{G}$ for Different Cuts Depth $d_{R}$. The Radiator Size is $a_{R}$ $=10 \mathrm{~mm}$ at $b_{R}=24 \mathrm{~mm}$

We note that both graphs are close and have two intervals of $B W$ values decrease and one interval of $B W$ values increase. The interval at which $B W$ values increase at the values of $b_{G}$ from $18 \mathrm{~mm}$ to $24-26 \mathrm{~mm}$ corresponds to the range where the values of $b_{G}$ become comparable with the radiator length $b_{R}$. At this interval, the time of the current path along the metal surface of the ground plane approaches the transit time of radiator current. At the same time, the minima on the alignment graphs of the antennas $\left(S_{11}\right.$ dependencies

$$
B W=\frac{V S W R-1}{Q \sqrt{V S W R}},
$$

where VSWR is the voltage standing wave ratio. $Q$ factor shows how many times the energy reserves in the system are greater than the losses, and it is proportional to the ratio of the stored energy and the radiated energy. In the case of a microstrip antenna ground plane and the radiator are the capacitor plates, in which the stored energy is proportional to the ground plane area (capacitor volume). Thus, the increase of the ground plane size $b_{G}$ leads to an increase in the stored energy. Since the radiated power does not change much, the antenna quality factor increases. The bandwidth is inversely proportional to the quality factor, and, consequently, the increase of the ground plane size leads to the narrowing of the bandwidth.

Thus, it is possible to draw the following conclusions regarding the effect of the ground plane dimensions on the bandwidth. When the length of the ground plane on frequency) approach each other, and the interval at which the reflection coefficient values are less than the specified value $\left(S_{11}<-5 \mathrm{~dB}\right)$ is increased. Note also that with the further increase of the ground plane, the base frequency begins to be determined by the length of the current path that passes through the ground plane (this occurs at $b_{G}>30 \mathrm{~mm}$ ).

Let us explain the intervals of $B W$ value decrease. It is known that the bandwidth of the antenna is inversely proportional to its quality factor:

and the radiator are significantly different, increase of the ground plane linear dimensions results in the decrease of the bandwidth. However, the maximum $B W$ values are reached when the ground plane and radiator length values are close to each other.

Then let us study the effect of the radiator dimensions $(R D)$ on the bandwidth. Fig. 7 shows the dependencies of $B W$ values on the radiator dimensions. The value of $R D=1$ corresponds to the unchanged antenna considered in the work. For example, with $R D=1.5$, all parameters of the radiator become one and a half times larger, at $R D=2$ they become twice as large, etc. Also on the figure below, the graph with black triangles corresponds to the antenna with the cutouts depth $d_{R}=2.5 \mathrm{~mm}$, the graph with white triangles corresponds to the antenna with $d_{R}=0.5 \mathrm{~mm}\left(d_{R}\right.$ values are given for $R D=1$ ). 


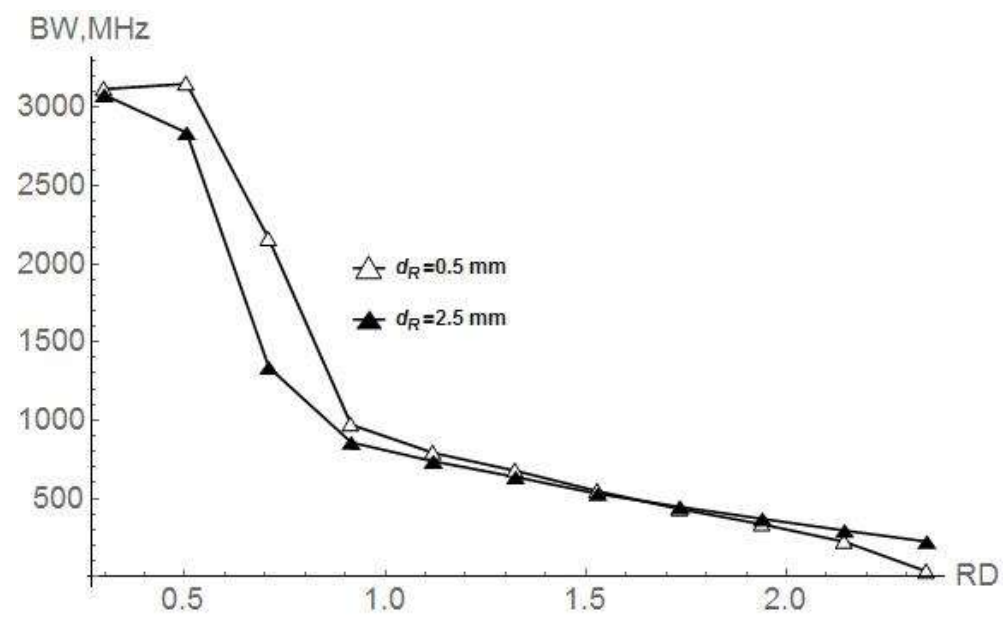

Figure 7. Dependence of BW on the Radiator Scale RD for Different Cuts Depth $d_{R}$. The Radiator Size is $a_{R}=10 \mathrm{~mm}$ at $b_{R}=24 \mathrm{~mm}$

From the analysis of the curves on Fig. 7 we can conclude that for small radiators $R D \leq 0.4$ the bandwidth is the largest one. When the scale is increased from 0.1 to 1.25 , the bandwidth is reduced drastically (in four times). There is an insignificant narrowing of the bandwidth with a further increase of scale. Also, as in the case of the ground plane size change, the decrease values of $B W$ is conditioned by the increase of the stored energy (metal area).

Thus, the small dimensions of the radiator give a wide bandwidth. However, it must be noted that the base frequency also decreases strongly with the radiator

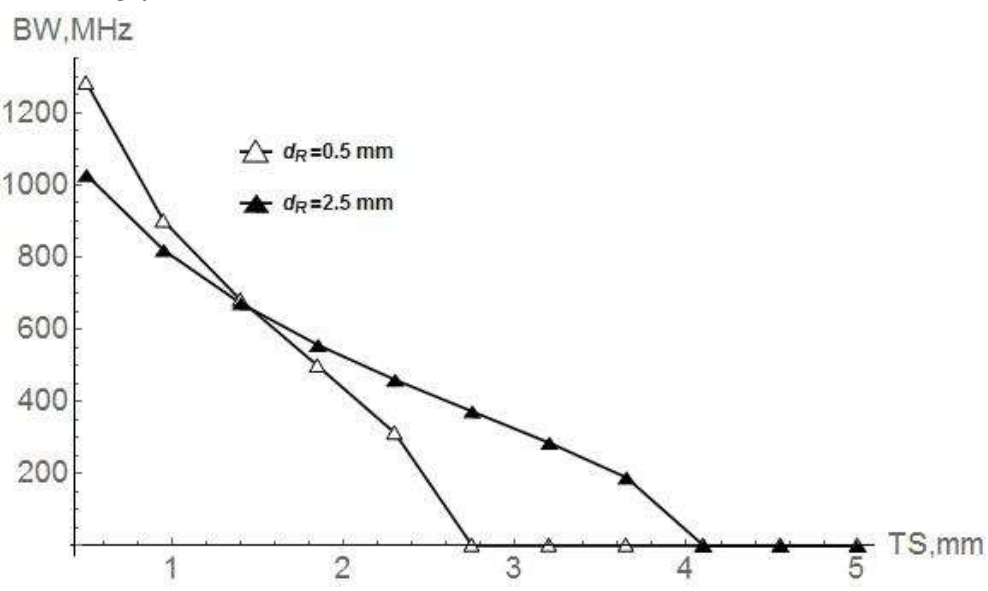

Figure 8. Dependence of BW on the Substrate Thickness TS for Different Cuts Depth $d_{R .}$ The Radiator Size is a $=10 \mathrm{~mm}$ at $b_{R}=24 \mathrm{~mm}$

Thus, it is preferable to choose the thickness of the radiator substrate no more than $1.5 \mathrm{~mm}$. It is also possible to explain the influence of the substrate layer thickness physically by increasing the capacitance of the capacitor. This leads to the increase of the quality factor and, accordingly, to the bandwidth decrease.

Let us note that the substrate in the antenna under consideration is homogeneous one, but choosing an inhomogeneous filling of the dielectric layer [27], it is possible to affect the bandwidth. The examples of the influence of a layer filling inhomogeneity with a dielectric material on the propagation of electromagnetic waves can be found in [28, 29]. It is increase [25]. Thus, the change in scale leads only to a slight increase of the fractional bandwidth.

The change of the substrate thickness on $B W$ is also used. For example, in [26], authors studied the influence of thickness and the filling of the substrate on the bandwidth. Let us consider (Fig. 8) the influence of the substrate thickness $(T S)$ on the bandwidth of our antenna. The increase of the substrate thickness results in a rapid decrease of $B W$ values. Moreover, for an antenna with a smaller cuts $\left(d_{R}=0.5 \mathrm{~mm}\right)$, the bandwidth decreases more rapidly, reaching a zero value at $T S \approx 2.8 \mathrm{~mm}$. 


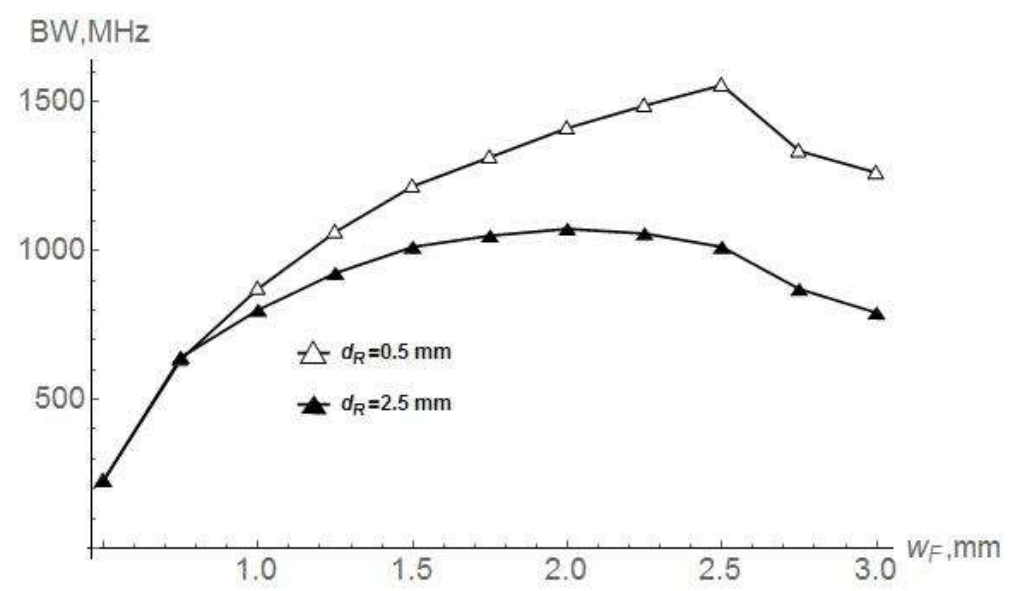

Figure 9. Dependence of $B W$ on the Feedline Width $w_{F}$ for Different Cuts Depth $d_{R}$. The Radiator Size is $a_{R}=10 \mathrm{~mm}$ at $b_{R}=\mathbf{2 4} \mathbf{m m}$

The analysis of the graphs shows that very thin feed lines give small $B W$ values, and the antennas with $w_{F}<$ $0.8 \mathrm{~mm}$ cannot be recommended because of the narrow bandwidth. Note that at $w_{F}=0.5 \ldots 2.4 \mathrm{~mm}$ we observe the increase of $B W$ values. Moreover, the bandwidth for the radiator with a cutouts size $d_{R}=0.5 \mathrm{~mm}$ is wider than for the radiator with the cutouts size $d_{R}=$ $2.5 \mathrm{~mm}$. The values $w_{F}$ from 1.5 to $2.5 \mathrm{~mm}$ give for the case $d_{R}=2.5 \mathrm{~mm}$ approximately the same bandwidth. While, for $d_{R}=0.5 \mathrm{~mm}$, the bandwidth grows in this interval.

Thus, it can be said that the influence of the feed line thickness on the antenna with a large cuts is less pronounced. It should also be noted that it is undesirable to use both narrow and wide feed lines.

\section{Conclusion}

The dependence of the bandwidth on various parameters of a microstrip antenna with the radiator of a symmetrical four-tooth shape was studied. It was shown that the bandwidth decreases with the radiator width increase. For a narrow radiator the bandwidth is reduced, when the cutouts is enlarged. For radiators whose length and width values are close, values of the bandwidth first increases, then decreases with the cuts increase.

When the length of the ground plane and the radiator are significantly different, then the increase of the ground plane linear dimensions results in the bandwidth decrease. However, the maximum bandwidth values are reached when the ground plane and radiator values become close to each other. The increase of the radiator dimensions leads to the bandwidth decrease. However, it should be noted that the base frequency also decreases. Thus, the change in scale leads only to a slight increase in the fractional bandwidth.

As the thickness of the substrate is increased, the bandwidth is narrowed. The effect of the feed line thickness on the antenna with a large cutouts is less pronounced.
Let us note that it is preferable to use the substrates with the thickness of up to $1.5 \mathrm{~mm}$, and it is undesirable to use too narrow and too wide feed lines.

\section{Summary}

The microstrip antenna with the radiator of symmetrical four-tooth shape is considered. The dependencies of the bandwidth at the base frequency on geometric parameters of the radiator and other antenna parameters were studied. The regression model is developed for the bandwidth. The behavior of the bandwidth is analyzed when other parameters of the antenna are changed.

The obtained regression model (together with the regression model for the base frequency) can be used to find the maximum values of $B W$ at given constraints on the radiator parameters. After the obtaining of the radiator dimensions, it is possible improve the bandwidth by the corresponding change of other antenna parameters.

\section{Acknowledgement}

The work is performed according to the Russian Government Program of Competitive Growth of Kazan Federal University.

\section{References}

[1] R. Garg, P. Bhartia, I. Bahl, A. Ittipiboon, Microstrip antenna design handbook. Artech house, 2001.

[2] T.A. Milligan, Modern antenna design. New Jersey, John Wiley \& Sons, 2005.

[3] R.M. Elsagheer, "Study on bandwidth enhancement techniques of microstrip antenna", Journal of Electrical Systems and Information Technology, vol. 3, pp. 527-531, 2016. doi: 10.1016/j.jesit.2015.05.003.

[4] A. Kumar, N. Gupta, P.C. Gautam, "Gain and bandwidth enhancement techniques in microstrip patch antennas - a review", International Journal of Computer Applications, 
vol. 148, no. 7, pp. 9-14, 2016. doi: 10.5120/ijca2016911207.

[5] C. Sharma, A. Sharma, "A review paper based on various bandwidth enhancements techniques for ultra-wide band antennas", International Journal of Science Technology \& Engineering, vol. 2, no. 8, pp. 1-7, 2016.

[6] M. Gouda, M.Y.M. Yousef, "Bandwidth enhancement techniques comparison for ultra wideband microstrip antennas for wireless application", Journal of Theoretical and Applied Information Technology, vol. 35, no. 2, pp. 184-193, 2012.

[7] S.S. Mishra, M.K. Singh, D.C. Dhubkariya, "Performance analysis and bandwidth enhancement of rectangular microstrip patch [MSP] antenna using compact double "L" slotted technique for broadband applications", International Journal of Enhanced Research in Science Technology $\backslash \&$ Engineering, vol. 3, no. 1, pp. 418-423, 2014.

[8] S.N. Ather, P.K. Singhal, "Truncated rectangular microstrip antenna with $\mathrm{H}$ and $\mathrm{U}$ slot for broadband", International Journal of Engineering Science and Technology, vol. 5, no. 1, pp. 114-118, 2013.

[9] D. Sugumar, T.J.S. Hephzibah, T.A. Jones, C.V. Viji, "E slotted rectangular microstrip antenna with coaxial feed for bandwidth enhancement", International Journal of Computer Science, Systems Engineering and Information Technology, vol. 4, no. 1, pp. 77-80, 2011.

[10] J.C. Rao, K.P. Rajashekar, G.P. Kumar, "H-U-E Shaped slotted microstrip antenna for bandwidth enhancement", International Journal of Future Generation Communication and Networking, vol. 7, no. 4, pp. 141-148, 2014.

[11] S.B. Pokharkar, S.P. Bhosale, "Improve bandwidth of microstrip slotted patch antenna using two square shape defected ground structure", International Journal of Advanced Research in Electrical, Electronics and Instrumentation Engineering, vol. 3, no. 6, pp. 9823-9828, 2014.

[12] K. Mondal, P. Sarkar, "Enhancement of the gain and bandwidth of the microstrip patch antenna with modified ground plane" International Journal of Microwave and Wireless Technologies, vol. 9, no. 5, pp. 1179-1184, 2017. doi: 10.1017/S1759078716001276.

[13] N. Prombutr, P. Kirawanich, P. Akkaraekthalin, "Bandwidth enhancement of UWB microstrip antenna with a modified ground plane", International Journal of Microwave Science and Technology, ID 821515, 7 pages, 2009. doi: $10.1155 / 2009 / 821515$.
[14] P. Mishra, P. Gupta, "Bandwidth improvement of UWB microstrip antenna using finite ground plane", International Journal of Engineering Research and Applications, vol. 5, no. 6, pp. 2225, 2015.

[15] R.C. Hadarig, M.E. de Cos, F. Las-Heras, "Microstrip patch antenna bandwidth enhancement using AMC/EBG structures", International Journal of Antennas and Propagation, ID 843754, 6 pages, 2012. doi:10.1155/2012/843754.

[16] S.M. Shaka, R.T. Prashant, R.M. Vani, P.V. Hunagund, "Enhanced bandwidth of rectangular microstrip antenna using uniplanar EBG cells", International Journal of Innovative Research in Computer and Communication Engineering, vol. 2, no. 4, pp. 3860-3865, 2014.

[17] H. Xiong, J.-S. Hong, Y.-H. Peng, "Impedance bandwidth and gain improvement for microstrip antenna using metamaterials", Radioengineering, vol. 21, no. 4, pp. 993-998, 2012.

[18] T. Bougoutaia, D. Khedrouche, A. Hocini, "Bandwidth improvement for compact microstrip patch antenna using metamaterials", Acta Physica Polonica A, vol. 129, no. 4, pp. 538-540, 2016. doi: 10.12693/APhysPolA.129.538.

[19] A.-A. Kalteh, S. Nikmehr, "Wide-band combshaped slotted microstrip patch antenna", 2nd National Electrical Eng. Conf., ID NEEC2010F-337, Febr. 2010.

[20] A.G. Markina, N.B. Pleshchinskii, D.N. Tumakov, "On electrical characteristics of comb-shaped microstrip antennas", Young Researchers in Electrical and Electronic Engineering (EIConRus), 2017 IEEE Conference of Russian, IEEE, pp. 179-183, 2017. doi: 10.1109/EIConRus.2017.7910523.

[21] A. Boutejdar, A. A. Ibrahim, E. P. Burte, "Novel microstrip antenna aims at UWB applications", Microwaves \& RF, pp. 62-66, Oct. 2015.

[22] K. Mandal, "Seven-Band Comb-Shaped Microstrip Antenna for Wireless Systems", PIER Letters, vol. 59, pp. 15-20, 2016.

[23] D.N. Tumakov, G.V. Abgaryan, D.E. Chickrin, P.A. Kokunin, "Modeling of the Koch-type wire dipole", Applied Mathematical Modelling, vol. 51, pp. 341-360, 2017. doi: 10.1016/j.apm.2017.07.007.

[24] J.O. Rawlings, S.G. Pantula, D.A. Dickey, Applied regression analysis: a research tool, Springer Science \& Business Media, 2001. 
[25] A.G. Markina, D.N. Tumakov, N.B Pleshchinskii, "On base frequency for the symmetrical four comb-tooth-shaped microstrip antenna", Journal of Fundamental and Applied Sciences, vol. 9, no. 1S, pp. 1534-1547, 2017. doi: 10.4314/jfas.v9i1s.802.

[26] L.C. Paul, Md.S. Hosain, S. Sarker, M.H. Prio, M. Morshed, A.K. Sarkar, "The effect of changing substrate material and thickness on the performance of inset feed microstrip patch antenna", American Journal of Networks and Communications, vol. 4, no. 3, pp. 54-58, 2015. doi: 10.11648/j.ajnc.20150403.16.

[27] N. Rao, D.V. Kumar, "Gain and bandwidth enhancement of a microstrip antenna using partial substrate removal in multiple-layer dielectric substrate", PIERS, pp. 1285-1289, 2011.

[28] A. Anufrieva, D. Tumakov, "Peculiarities of electromagnetic wave propagation through layers with ridge-shaped refractive index distribution", MMET, pp. 386-389, 2012. doi: 10.1109/MMET.2012.6331200.

[29] N.B. Pleshchinskii, D.N. Tumakov, "Analysis of electromagnetic wave propagation through a layer with graded-index distribution of refraction index", PIERS, pp. 425-429, 2012.

[30] N.B. Pleshchinskii, D.N. Tumakov, "The reconstruction of dielectric profile of a layer for the harmonic wave case", PIERS, pp. 643-647, 2013.

[31] D. Tumakov, "On optimal frequencies for reconstruction of a one-dimensional profile of gradient layer's refractive index", International Journal of Optics, ID 841960, 7 pages, 2014. doi:10.1155/2014/841960.

[32] M.S. Rabbani, H. Ghafouri-Shiraz, "Simple methods for enhancing bandwidth of a rectangular microstrip patch antenna", 2nd IET Annual Active and Passive RF Devices Seminar, pp. 1-4, 2014. doi: 10.1049/ic.2014.0184. 